\title{
NEW NERVE CUFF ELECTRODE BASED ON A SHAPE MEMORY ALLOY ARMATURE
}

\author{
M-A. Crampon, M. Sawan, V. Brailovski*, F.Trochu* \\ Department of Electrical \& Computer Engineering, *Department of Mechanical Engineering, \\ Ecole polytechnique de Montreal, P.O.Box 6079, Station Centre-Ville, Montreal, Qc, Canada H3C 3A7 \\ Fax: 514340 4147, E-mail: crampon@vlsi.polymtl.ca
}

\begin{abstract}
In this paper, we present a new nerve cuff electrode based on a shape memory alloy (SMA) armature. This device is dedicated to functional electrical stimulation of the bladder in spinal cord injured patients. The SMA armature performs the closing of the electrode, making its installation around the nerve much easier, quicker and safer. Both remarkable mechanical properties of SMA materials namely shape memory effect and superelasticity, can be used to obtain the desired actuation of the electrode closing. The manufacturing procedure of this new electrode is described. It does not require any expensive or complex techniques. Bipolar and tripolar electrodes have been manufactured with an inner diameter of $1.6 \mathrm{~mm}$ and a cuff wall thickness of $0.8 \mathrm{~mm}$. Acute studies in dogs are being carried out to validate the device and the implantation procedure. In order to test the electrical functionality of the electrode, a fully programmable biphasic stimuli generator has been designed and implemented using FPDs (Field Programmable Devices).
\end{abstract}

\section{INTRODUCTION}

Over the last two decades, nerve cuff electrodes have been used in many neuroprosthetic applications such as motor activation of the lower extremity $[1,2]$, bladder incontinence and retention control [3,4], treatment of chronic pain [5], electroneurograms recordings [6], etc. If can be employed, nerve cuff electrodes are generally preferred to muscular electrodes since they do not risk to cause any mechanically induced muscular damage during activation. They also enable the activation of several muscles through a single nerve if an appropriate stimulus is transmitted. For these reasons, they offer an accurately reliable and flexible interface between the stimulator and the biological tissue. Among the nerve cuff electrodes, two main types can be distinguished: splitcylinder and spiral cuff electrodes. The split-cylinder cuff electrodes [7] are made of a medical grade silicone rubber cylinder cuff with metallic electrical contacts on its inner side. The cuff is generally secured around the nerve with sutures. The spiral cuff electrodes [8] are made of a selfcurling sheath of silicone rubber with embedded conductive segments. They do not need any suture to be fixed since they self-wrap tightly around the nerve. The major problem with the electrodes just described is their difficulty to be manipulated and installed around the nerve due to their small dimensions and the restrained area available for implantation. The use of a Shape Memory Alloy (SMA) structure for closing the electrode enables to overcome these difficulties. These materials are used in a large spectrum of applications, from electrical engineering and aeronautics to biomedicine. The biomedical devices using SMA, such as cardiovascular stents, are quite an ongoing research field [9]. The SMA armature technique has already been experimented by Niemi and al. [10] for another type of nerve electrode but the device manufacturing requires complex and expensive laser technologies.

In the present paper, we report the design and manufacturing of a new split-cylinder nerve cuff electrode that can be easily and quickly installed around the nerve. Its closing is performed by a shape memory alloy structure embedded in the silicone nubber cylinder cuff.

\section{METHODS AND MATERIALS}

\section{A. General description.}

The new nerve cuff electrode with SMA armature is presented on Figure 1. Its design is based on classic splitcylinder cuff electrodes, but a SMA armature has been added inside the cuff wall. This SMA structure provides to the whole electrode new mechanical properties. It enables the electrode to close by itself around the nerve and to be maintained in place without requiring any external fixation

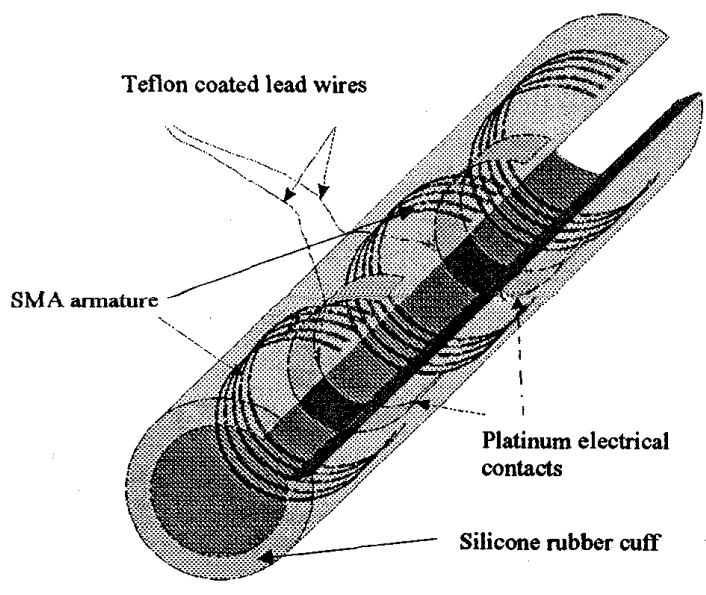

Fig.1: The nerve cuff electrode with SMA armature. 
means such as sutures. The electrode cuff is molded in silicone rubber. Electrode contacts are $1 \mathrm{~mm}$ large platinum foil bands. In order to connect the electrode to the external or implanted stimulator or recorder, lead wires are welded onto the platinum contacts. These are multi-strands stainless steel wires coated with Teflon®. The SMA armature is made of medical grade NiTi wires of $0.1 \mathrm{~mm}$ of diameter.

\section{B. Remarkable properties of shape memory alloys.}

SMA are well known and papers or books describing their technology are numerous [11-13] but a short description will be done here. In order to understand the mechanical behavior of the electrode, we have to point out the two remarkable properties of SMA materials: the shape memory effect and the superelasticity

The shape memory effect happens when a SMA material deformed at a certain temperature is heated to a higher temperature. It then recovers the specific shape it has previously memorized. The shape recovery temperature is noted $A_{f}$ because it corresponds to the finishing austenitic phase transformation of the alloy. The material can memorize any desired shape by undergoing a specific thermal treatment over $400^{\circ} \mathrm{C}$ for several hours. And by adjusting the thermal treatment duration, the $A_{f}$ temperature can be regulated at the desired value.

Above the $A_{f}$ temperature, the alloy becomes superelastic. This means that, over a certain amount of mechanical stress, the material can be easily and reversibly strained up to $8 \%$ or more. Under low stress, the material Young's modulus - stress to strain ratio during elastic deformation - is similar to the one of any metal but under higher stress, it becomes as low as the one of a rubber band.

\section{Electrode armature behavior}

Considering the SMA materials properties, we have two possibilities for designing the cuff electrode. We can either use a shape memory armature or a superelastic armature. As described on Figures 2 and 3 , the way of installing the electrode around the nerve will depend on the selected design method.

If a shape memory armature is to be used, then the alloy $A_{f}$ temperature will be fixed slightly under $37^{\circ} \mathrm{C}$. At ambient temperature, the electrode is initially closed (Fig.2a). By cooling the electrode around $10^{\circ} \mathrm{C}$, the surgeon can easily open the cuff and the electrode will remain in open position even if is taken back to ambient temperature (Fig.2b). In this

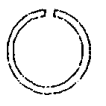

a)

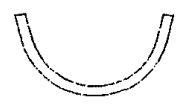

b)

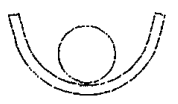

c)

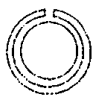

d)
Fig.2: Installation procedure of a cuff electrode with a shape memory armature. a) Electrode at ambient temperature, b) Electrode opened by the physician at $10^{\circ} \mathrm{C}, \mathrm{c}$ ) Placing of the electrode around the nerve, d) Self-closing of the cuff at $37^{\circ} \mathrm{C}$. opened configuration, the electrode can be easily placed under the it nerve (Fig.2c). In the biological environment, the SMA armature warms up to its $A_{f}$ temperature and then recovers its initial shape, closing the electrode (Fig.2d). When closed around the nerve, the SMA armature makes the cuff rigid enough to insure the cuff mechanical stability.

If a superelastic armature is to be used, the $A$ temperature is fixed below ambient temperature. At room temperature, the electrode is initially closed (fig.3a). The physician pulls apart the two edges of the electrode cuff, strongly enough to reach the SMA material superelastic state. Then, the electrode cuff opens easily (fig.3b). Keeping the cuff opened, the physician can place the electrode near the nerve. When he slackens the cuff, the electrode comes to close elastically around the nerve (fig.3c). The installed electrode is once again rigid enough to remain stable on the nerve.

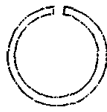

a)

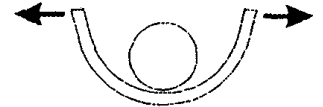

b)

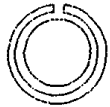

Fig.3: Installation procedure of a cuff electrode with a superelastic armature. a) Closed electrode at room temperature, b) Electrode opened by the surgeon at room temperature, $c$ ) Elastic closing of the electrode around the nerve.

\section{Electrode fabrication.}

\section{Used materials and biocompatibility.}

The electrode-tissue contact has to be exclusively made of biocompatible materials. The electrode cuff is made of Silastic $B$ and electrode contacts are cut from a $0.025 \mathrm{~mm}$ thick platinum foil. The lead wires are multi-strands stainless steel wires coated with Teflon $ß$ (e.g. Cooner Wire, AS634). All these materials are commonly used in biomedical applications and are completely biocompatible. For the SMA armature, medical grade NiTi wires of $0.1 \mathrm{~mm}$ of diameter are used (Shape Memory Applications, inc.). This shape memory alloy $(50.7 \%$ Nickel, $49.3 \%$ Titanium) is biocompatible and has already been used in different biomedical applications such as cardiovascular stents [9]. Nevertheless, it is still undergoing acute long term biocompatibility testing. For this reason and also because the armature needs to be electrically isolated, the electrode SMA structure is completely embedded in Silastic $($.

\section{Armature design.}

As shown on Figure 1, for a bipolar electrode, the armature is composed of three sets of NiTi wire split rings. A tripolar electrode would have an armature with four sets of split rings. In order to give the NiTi this final shape, it is wound into a spring on a metallic rod of $1.9 \mathrm{~mm}$ of diameter. It is then thermally treated at $470^{\circ} \mathrm{C}$ during one to three hours depending on the type of mechanical behavior (shape memory effect or superelasticity) we want to obtain. The 
treated SMA spring is covered by a first thin layer of Silastic(8) in order to create an additional cohesion between the different spring turns. Then, we can cut the spring longitudinally in order to obtain the armature elements presented on Figure 4.

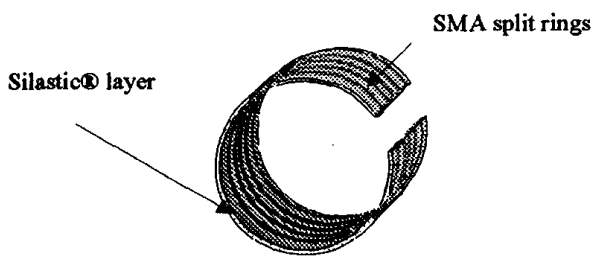

Fig.4: SMA armature element composed by several split rings of NiTi wire maintained by a thin layer of Silastic $\$$.

\section{Electrode assembling.}

The electrode is assembled on a stainless steel cylinder of $1.6 \mathrm{~mm}$ of diameter according to the method of M. Haugland [7]. The armature elements are placed in between the electrode platinum contacts. They are isolated from the internal side of the cuff by Silastic $B$. When all the armature elements and contacts are mounted on the cylinder, they are deep coated in a low viscosity Silastic $\gg$ and heptane solution in order to obtain a cuff of minimal thickness.

\section{Testing procedure.}

In order to validate the new closing method and the overall device efficiency, the electrodes have to undergo in vitro and in vivo testing.

In vitro testing.

Before implantation, the exact electrode closing at body temperature is verified in regulated temperature water. Secondly, acute mechanical testing and simulations are undertaken in order to evaluate the cuff rigidity and the maximum traction stress that can be applied on the lead wires before the cuff releases from the nerve.

In vivo testing.

In order to evaluate the efficiency of the proposed electrodes, in vivo acute and chronic studies are being carried out on several samples. In acute experimentation, the implantation facility and quickness as well as the electrical efficiency of the new electrodes can be tested. Then, short to long term mechanical stability and biocompatibility will be evaluated in chronic studies.

To perform electrical testing in stimulation mode, we have developed a dedicated fully programmable stimuli generator. This generator is implanted on a FPGA (Field Programmable Gate Array) component. Its block diagram is shown on Figure 5a. It provides a biphasic ramp stimulus made of two signals of independent frequency (Figure $5 b$ ). This type of stimulus causes a selective stimulation of the sacral root in order to achieve low pressure voiding of the bladder. The stimulation parameters are programmed by the user from the parallel port of a personal computer.

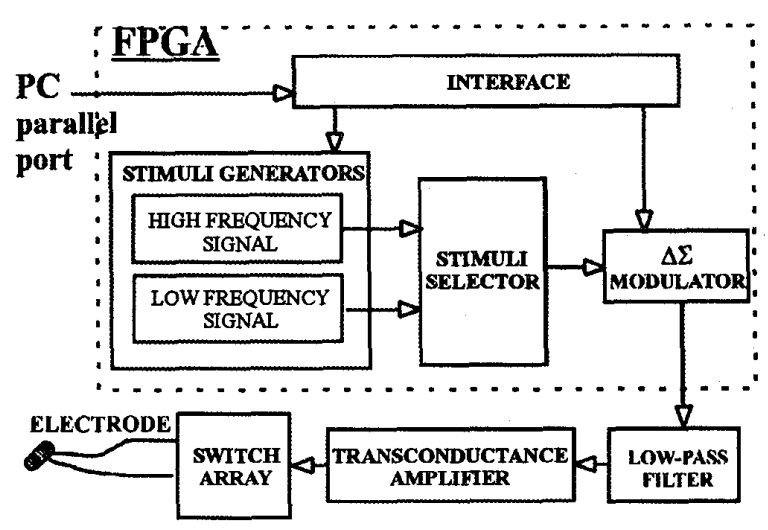

a)

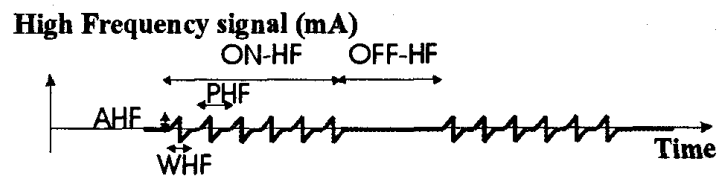

Low Frequency signal (mA)

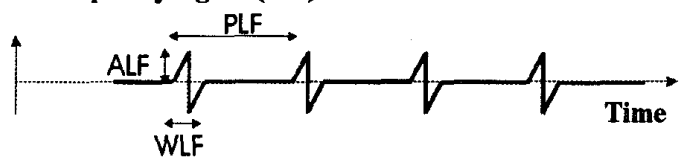

Stimulus (mA)

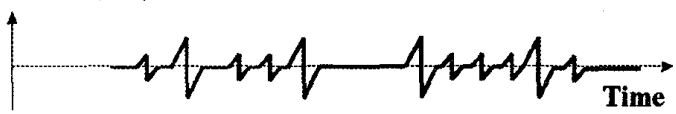

b)

Fig.5: The stimuli generator. a) The block diagram of the FPGA implantation, b) A typical stimulus waveform.

\section{PRELIMINARY RESULTS}

Different types of electrodes have been fabricated in order to be implanted on sacral root of dogs: bipolar electrodes for functional electrical stimulation of the bladder and tripolar electrodes for electroneurograms (ENG) recording. For both types, electrodes with memory effect armature and others with superelastic armature have been completed. The shape recovery temperature of the SMA material is fixed at $35^{\circ} \mathrm{C}$ in the first case and at $10^{\circ} \mathrm{C}$ in the second case. The electrode cuffs are $1.6 \mathrm{~mm}$ of diameter and about $0.8 \mathrm{~mm}$ of thickness. Their lengths are $12 \mathrm{~mm}$ for bipolar electrodes and $15 \mathrm{~mm}$ for tripolar ones. These dimensions are adequate for implantation on S2 sacral root of 
Table 1: Stimulation parameter range.

\begin{tabular}{|l|c|c|c|c|c|c|c|c|c|c|}
\hline & \multicolumn{9}{|c|}{ High Frequency } & \multicolumn{4}{c|}{ Low Frequency } \\
\hline Parameter & Amplitude & Period & Pulse Widkh & Train length & Trains spacing & Amplitude & Period & Pulse Width & Train length & Trains spacing \\
\hline Symbol & AHF & PHF & WHF & ON-HF & OFF-HF & ALF & PLF & WLF & ON-LF & OFF-LF \\
\hline Units & mA & ms & $\mu s$ & Nb of pulses & Nb of pulses & mA & ms & $\mu s$ & Nb of pulses & Nb of pulses \\
\hline Range & $0-2$ & $1-10$ & $0-500$ & $0-100$ & $0-100$ & $0-2$ & $10-100$ & $0-500$ & $0-100$ & $0-100$ \\
\hline
\end{tabular}

dogs. A thicker cuff wall could lead to tissue damage in the nerve neighborhood due to mechanical friction. Moreover, the electrode cuff cannot be longer than $2 \mathrm{~cm}$ because of the restricted nerve length exposed during the surgery. S2 sacral root of the dogs has a diameter of $1.05 \pm 0.15 \mathrm{~mm}$ [14]. The cuff inner diameter is then about 1.5 times as large as the considered nerve diameter. This respects the AAMI recommendation for safe cuff electrode use [15].

As expected, the electrodes with superelastic armature are closed at ambient temperature and can be easily manipulated and opened by pulling the edges of the cuff apart. And as soon as the stress is released, the cuff comes to close again. The behavior of the electrodes with shape memory armature is slightly different of the one predicted (see Figure 2). They almost behave as the electrodes with superelastic armature because of the high elasticity of the silicone cuff. The electrode happens to be difficult to be kept opened even at low temperature because the silicone cuff spring back force is high compared to the armature rigidity. The shape memory design rigidity has to be improved by increasing the number of SMA rings in the different sets of the armature.

The stimuli generator shows satisfying operating. The different parameters can be easily changed before each stimulation within a wide range as presented on Table 1.

Acute experimentation on dogs has started recently and our results will be reported soon.

\section{DISCUSSION}

The armature rigidity has to be carefully chosen. We have experimented that a low rigidity shape memory armature does not lead to a correct behavior of the whole electrode. Inversely, a too rigid armature could tear the silicone cuff during closing. The armature rigidity can be evaluated by simulating the SMA material mechanical behavior. Mechanical testing of the NiTi wire is presently undertaken to determinate the material characteristics that are necessary for running the simulation software.

\section{CONCLUSION}

We have described a new type of nerve cuff electrode activated by a SMA armature. It is easier to install on the nerve than available nerve electrodes. It is also fabricated at low cost without requiring any complex technique. The superelastic armature design seems to be more promising than the one with a shape memory armature. Acute and chronic studies are undertaken in animals (dogs) to evaluate the electrode mechanical behavior and biocompatibility. In the future, we will consider electrode cuffs of smaller diameter that could extend in case of nerve diameter increase.

\section{ACKNOWLEDGMENTS}

Authors would like to acknowledge the financial support from the Natural Sciences and Engineering Research Council of Canada (NSERC) and the International Council for Canadian Studies.

\section{REFERENCES}

[1] C. Veraart, W. M. Grill, and J.T. Mortimer, "Selective Control of Muscle Activation with a mulpipolar Nerve Cuff Electrode", IEEE Trans. Biomed. Eng., vol. 40, pp. 640-653, July 1993.

[2] R. L. Waters, D. R. McNeal, W. Faloon, and B. Clifford, "Functional electrical stimulation of the peroneal nerve for hemiplegia: Long term clinical follow-up", J. Bone Joint Surg., vol. 67-A, pp. 792-793, 1985.

[3] M. Sawan et al., "Stimulator Design and Subsequent Stimulation Parameter Optimization for Controlling Micturition and Reducing Urethral Resistance", IEEE Trans. Rehab. Eng., vol. 4, No. 1, March, pp. 39-46, 1996.

[4] R. A schmidt, H. Bruschini and E. A. Tanagho, "Feasability of inducing micturition through chronic stimulation. of sacral roots", Urol., vol. 12, pp. 471-477, 1978.

[5] B. S. Nashold, J. L. Goldner, J. B. Mullen, and D. S. Bright, "Longterm pain control by direct peripheral nerve stimulation", J. Bone Joint Surg., vol. 64-A, pp. 1-10, 1982

[6] J. E. Loeb, R. A Peck, "Cuff electrodes for chronic stimulation and recording of peripheral nerve activity", J. Neuroscience Methods, vol. 64, pp. 95-103, 1996.

[7] Morten Hlaugland, "A Flexible Method for Fabrication of Nerve Cuff Electrodes", IEEE/EMBS Proceedings, Amsterdam, 1996.

[8] G.G. Naples, J.T. Mortimer, A. Scheiner, J.D. Sweeney, "A Spiral Nerve Cuff Électrode for Peripheral Nerve Stimulation", IEEE Transaction on Biomedical Eng., vol. 35, no. 11, p. 905-915, nov. 1988.

[9] I. Yu. Khmelevskaya et al., "Application of Ni-Ti SME Alloys to XRay Endslenting and Other Medical Fields", Proceedings of the First International Conference on Shape Memory and Superelastic Technologies, Asilomar, Ca., USA, 1994.

[10] J.B.Niemi, J.D.Harry, "Stabilization and Insertion of Peripheral nerve Electrodes Using a Ni-Ti Cuff", Proceedings of the First International Conference on Shape Memory and Superelastic Technologies, Asilomar, Ca., USA, 1994.

[11] H. Funakudo, "Shape Memory Alloys", Gordon \& Breach, Amsterdam 1987.

[12] "Engineering Aspects of Shape Memory Alloys", ed. T. Duerig et al.) Butterworth-Heinemann, 1990.

[13] E. Patoor, M. Berveillen, "Technologie des alliages à mémoire de forme ", Hremes, Paris, 1994.

[14] J. N. Peck, J. S. Walter, D. F. Merkley, and M. H. Greer, "Feasability of Implant Driven Micturation in Paraplegic Dogs", Veterinary Surgery, vol. 26, pp. 33-44, 1997.

[15] Association for the Advancement of Medical Instrumentation, "American National Standard for Implantable Peripheral Nerve Stimulators", 1984. 